\title{
Erratum to: Partial regularity of stable solutions to the Emden equation
}

\section{Kelei Wang}

Published online: 17 November 2012

(C) Springer-Verlag Berlin Heidelberg 2012

\section{Erratum to: Calc. Var. \\ DOI 10.1007/s00526-011-0446-3}

Recently the authors of [1] pointed out that the proof of Lemma 3.2 in [2] is incorrect. ${ }^{1}$ The following is the corrected formulation and proof.

Lemma $0.1 \exists \varepsilon_{0}>0, \theta \in(0,1)$, which depend only on the dimension $n$, such that for $a$ stable solution $u$ of $-\Delta u=e^{u}$ in $B_{2}(0)$, if

$$
2^{2-n} \int_{B_{2}(0)} e^{u}=\varepsilon,
$$

where $\varepsilon \leq \varepsilon_{0}$, then

$$
\theta^{2-n} \int_{B_{\theta}(0)} e^{u} \leq \frac{1}{2} \varepsilon .
$$

We can iterate (0.2) to get a bound of $\left\|e^{u}\right\|_{M^{n / 2+\delta\left(B_{\theta}(0)\right)}}$ for some $\delta>0$ (depending only on $\theta$ ). Then we get the smoothness of $u$ in $B_{\theta / 2}(0)$ as before. Note that, by the Hölder inequality, the assumption of Theorem 3.1 in [2] implies that $u$ satisfies (0.1).

1 I would like to thank Louis Dupaigne for explaining their results to me. Their method can be used to give another proof of the partial regularity result in [2].

The online version of the original article can be found under doi:10.1007/s00526-011-0446-3.

K. Wang $(\varangle)$

School of Mathematics and Statistics, The University of Sydney, Sydney, NSW 2006, Australia

e-mail: keleiw@mail.usyd.edu.au

Present Address:

K. Wang

Wuhan Institute of Physics and Mathematics, Chinese Academy of Sciences, Wuhan 430071, China

e-mail: wangkelei@wipm.ac.cn 
Proof Take a function $\eta \in C_{0}^{\infty}\left(B_{2}(0)\right)$, such that $0 \leq \eta \leq 1$, and $\eta \equiv 1$ in $B_{1}(0)$. By Farina's estimate (i.e. taking $\alpha=1 / 2$ in the estimate Eq. (2.1) in [2]), we have

$$
\int e^{2 u} \eta^{2} \leq C \int e^{u}\left(|\nabla \eta|^{2}+\left|\Delta \eta^{2}\right|\right)
$$

Hence $\left\|e^{u}\right\|_{L^{2}\left(B_{1}(0)\right)} \leq C \varepsilon^{1 / 2}$.

Take the decomposition $u=v+w$ in $B_{1}(0)$, where $v$ satisfies

$$
\begin{cases}-\Delta v=e^{u}, & \text { in } B_{1}(0) \\ v=0, & \text { on } \partial B_{1}(0)\end{cases}
$$

Since $\|v\|_{L^{1}\left(B_{1}(0)\right)} \leq C \varepsilon$ (cf. Lemma 3.4 in [2]) and $\left\|e^{u}\right\|_{L^{2}\left(B_{1}(0)\right)} \leq C \varepsilon^{1 / 2}$, by the global $W^{2,2}$ estimate, $\|v\|_{W^{2,2}\left(B_{1}(0)\right)} \leq C \varepsilon^{1 / 2}$. Then by the Sobolev embedding theorem, $\|v\|_{L^{\frac{2 n}{n-4}\left(B_{1}(0)\right)}} \leq C \varepsilon^{1 / 2}$. By interpolation between $L^{q}$ spaces, we obtain

$$
\|v\|_{L^{2}\left(B_{1}(0)\right)} \leq\|v\|_{L^{1}\left(B_{1}(0)\right)}^{\frac{4}{n+4}}\|v\|_{L^{\frac{2 n}{n-4}\left(B_{1}(0)\right)}}^{\frac{n}{n+4}} \leq C \varepsilon^{\alpha}
$$

where $\alpha=\frac{n+8}{2 n+8}>1 / 2$. Then by interpolation between Sobolev spaces, we get

$$
\|\nabla v\|_{L^{2}\left(B_{1}(0)\right)} \leq \varepsilon^{\frac{1}{4}\left(\alpha-\frac{1}{2}\right)}\left\|\nabla^{2} v\right\|_{L^{2}\left(B_{1}(0)\right)}+C \varepsilon^{-\frac{1}{4}\left(\alpha-\frac{1}{2}\right)}\|v\|_{L^{2}\left(B_{1}(0)\right)} \leq C \varepsilon^{\beta},
$$

where $\beta>1 / 2$ depends only on $n$. From this we get

$$
\int_{B_{1}(0)} v e^{u}=\int_{B_{1}(0)}-v \Delta v=\int_{B_{1}(0)}|\nabla v|^{2} \leq C \varepsilon^{2 \beta} .
$$

We decompose the estimate of $r^{2-n} \int_{B_{r}(0)} e^{u}$ into two parts: $\left\{v \leq \varepsilon^{\gamma}\right\}$ and $\left\{v>\varepsilon^{\gamma}\right\}$, where $\gamma=\frac{1}{2}(2 \beta-1)>0$.

The first part can be estimated as in [2] (by using the fact that $e^{w}$ is subharmonic and $w<u$ in $B_{1}(0)$, see Lemma 3.3 in [2])

$$
\begin{aligned}
r^{2-n} \int_{B_{r}(0) \cap\left\{v \leq \varepsilon^{\gamma}\right\}} e^{u} \leq r^{2-n} \int_{B_{r}(0) \cap\left\{v \leq \varepsilon^{\gamma}\right\}} e^{\varepsilon^{\gamma}} e^{w} & \leq r^{2} e^{\varepsilon^{\gamma}} r^{-n} \int_{B_{r}(0)} e^{w} \\
& \leq r^{2} e^{\varepsilon^{\gamma}} \int_{B_{1}(0)} e^{u} \\
& \leq C r^{2} \varepsilon
\end{aligned}
$$

The second part can be estimated using $(0.5)$

$$
r^{2-n} \int_{B_{r}(0) \cap\left\{v>\varepsilon^{\gamma}\right\}} e^{u} \leq \varepsilon^{-\gamma} r^{2-n} \int_{B_{r}(0)} v e^{u} \leq C r^{2-n} \varepsilon^{2 \beta-\gamma} .
$$

Putting these together we get

$$
r^{2-n} \int_{B_{r}(0)} e^{u} \leq C r^{2} \varepsilon+C r^{2-n} \varepsilon^{2 \beta-\gamma}
$$


Note that $2 \beta-\gamma>1$. We can first choose $r=\theta$ small enough, then $\varepsilon_{0}$ small enough, such that for any $\varepsilon<\varepsilon_{0}$

$$
C \theta^{2} \varepsilon+C \theta^{2-n} \varepsilon^{2 \beta-\gamma} \leq \frac{1}{2} \varepsilon
$$

By this choice we get (0.2).

\section{References}

1. Dupaigne, L., Goubet, O., Warnault, G., Ghergu, M.: The Gel'fand problem for the biharmonic operator, arXiv: $1207.3645 \mathrm{v} 2$.

2. Wang, K.: Partial regularity of stable solutions to the Emden equation. Calc. Var. 44, 601-610 (2012) 